\title{
DIFFERENTIAL UNIFORMITY AND SECOND ORDER DERIVATIVES FOR GENERIC POLYNOMIALS
}

\author{
YVES AUBRY AND FABIEN HERBAUT
}

\begin{abstract}
For any polynomial $f$ of $\mathbb{F}_{2^{n}}[x]$ we introduce the following characteristic of the distribution of its second order derivative, which extends the differential uniformity notion:

$$
\delta^{2}(f):=\max _{\substack{\alpha \in \mathbb{F}_{2}^{*}, \alpha^{\prime} \in \mathbb{F}_{2}^{*}, \beta \in \mathbb{F}_{2^{n}} \\ \alpha \neq \alpha^{\prime}}} \sharp\left\{x \in \mathbb{F}_{2^{n}} \mid D_{\alpha, \alpha^{\prime}}^{2} f(x)=\beta\right\}
$$

where $D_{\alpha, \alpha^{\prime}}^{2} f(x):=D_{\alpha^{\prime}}\left(D_{\alpha} f(x)\right)=f(x)+f(x+\alpha)+f\left(x+\alpha^{\prime}\right)+$ $f\left(x+\alpha+\alpha^{\prime}\right)$ is the second order derivative. Our purpose is to prove a density theorem relative to this quantity, which is an analogue of a density theorem proved by Voloch for the differential uniformity.
\end{abstract}

Keywords: Differential uniformity, Galois closure of a map, Chebotarev density theorem.

Mathematics Subject Classification: 14G50, 11T71, 94A60.

\section{INTRODUCTION}

For any polynomial $f \in \mathbb{F}_{q}[x]$ where $q=2^{n}$, and for $\alpha \in \mathbb{F}_{q}^{*}$, the derivative of $f$ with respect to $\alpha$ is the polynomial $D_{\alpha} f(x)=f(x+\alpha)+f(x)$. The differential uniformity $\delta(f)$ of $f$ introduced by Nyberg in [6] is then defined by

$$
\delta(f):=\max _{(\alpha, \beta) \in \mathbb{F}_{q}^{*} \times \mathbb{F}_{q}} \sharp\left\{x \in \mathbb{F}_{q} \mid D_{\alpha} f(x)=\beta\right\} .
$$

To stand against differential cryptanalysis, one wants to have a small differential uniformity (ideally equal to 2 ). Voloch proved that most polynomials $f$ of $\mathbb{F}_{q}[x]$ of degree $m \equiv 0,3(\bmod 4)$ have a differential uniformity equal to $m-1$ or $m-2$ (Theorem 1 in [11]).

When studying differential cryptanalysis, Lai introduced in [5] the notion of higher order derivatives. The higher order derivatives are defined recursively by $D_{\alpha_{1}, \ldots, \alpha_{i+1}} f=D_{\alpha_{1}, \ldots, \alpha_{i}}\left(D_{\alpha_{i+1}} f\right)$, and a new design principle is given in [5]: "For each small $i$, the nontrivial $i$-th derivatives of function should take on each possible value roughly uniform". After considering the differential uniformity, it seems natural to investigate the number of solutions of the equation $D_{\alpha_{1}, \alpha_{2}} f(x)=\beta$, that is of the equation

$$
f(x)+f\left(x+\alpha_{1}\right)+f\left(x+\alpha_{2}\right)+f\left(x+\alpha_{1}+\alpha_{2}\right)=\beta
$$


and thus to consider the second order differential uniformity of $f$ over $\mathbb{F}_{q}$ :

$$
\delta^{2}(f):=\max _{\substack{\alpha \in \mathbb{F}_{q}^{*}, \alpha^{\prime} \in \mathbb{F}_{q}^{*}, \beta \in \mathbb{F}_{q} \\ \alpha \neq \alpha^{\prime}}} \sharp\left\{x \in \mathbb{F}_{q} \mid D_{\alpha, \alpha^{\prime}}^{2} f(x)=\beta\right\} .
$$

For example, the inversion mapping from $\mathbb{F}_{q}$ to itself which sends $x$ to $x^{-1}$ if $x \neq 0$ and 0 to 0 (and which corresponds to the polynomial $f(x)=x^{q-2}$ ) has a differential uniformity $\delta(f)=2$ for $n$ odd and $\delta(f)=4$ for $n$ even (see [6]). We will prove in Section 8 that it has a second order differential uniformity $\delta^{2}(f)=8$ for any $n \geqslant 6$.

The purpose of the paper is to prove that, as Voloch proved it for the differential uniformity, most polynomials $f$ have a maximal $\delta^{2}(f)$. More precisely, we prove (Theorem 7.1) that: for a given integer $m \geqslant 7$ such that $m \equiv 0(\bmod 8)($ respectively $m \equiv 1,2,7(\bmod 8))$, and with $\delta_{0}=m-4$ (respectively $\delta_{0}=m-5, m-6, m-3$ ) we have

$$
\lim _{n \rightarrow \infty} \frac{\sharp\left\{f \in \mathbb{F}_{2^{n}}[x] \mid \operatorname{deg}(f)=m, \delta^{2}(f)=\delta_{0}\right\}}{\sharp\left\{f \in \mathbb{F}_{2^{n}}[x] \mid \operatorname{deg}(f)=m\right\}}=1 .
$$

We follow and generalize the ideas of Voloch in [11. Let us present the strategy.

- In Section 2, we associate to any integer $m$ an integer $d$ depending on the congruence of $m$ modulo 4 (Definition 2.1). Then, if $\alpha$ and $\alpha^{\prime}$ are two disctinct elements of $\mathbb{F}_{q}^{*}$, we associate (Proposition 2.2) to any polynomial $f \in \mathbb{F}_{q}[x]$ of degree $m$ a polynomial $L_{\alpha, \alpha^{\prime}}(f)$ (which will be sometimes denoted by $g$ for simplicity) of degree less than or equal to $d$ such that:

$$
D_{\alpha, \alpha^{\prime}}^{2} f(x)=g\left(x(x+\alpha)\left(x+\alpha^{\prime}\right)\left(x+\alpha+\alpha^{\prime}\right)\right) .
$$

- In Section 3, we determine the geometric and the arithmetic monodromy groups of $L_{\alpha, \alpha^{\prime}}(f)$ when this polynomial is Morse (Proposition 3.1). For $\alpha$ and $\alpha^{\prime}$ fixed, we give an upper bound depending only on $m$ and $q$ for the number of polynomials $f$ of $\mathbb{F}_{q}[x]$ of degree at most $m$ such that $L_{\alpha, \alpha^{\prime}}(f)$ is non-Morse (Proposition 3.2).

- Section 4 is devoted to the study of the monodromy groups of $D_{\alpha, \alpha^{\prime}}^{2} f$. In order to apply the Chebotarev's density theorem (Theorem 5.1) we look for a condition of regularity, that is a condition for $\mathbb{F}_{q}$ to be algebraically closed in the Galois closure of the polynomial $D_{\alpha, \alpha^{\prime}}^{2} f(x)$ (Proposition 4.6).

- In Section 5, we use the Chebotarev theorem to prove that (Proposition 5.2) for $q$ sufficiently large and under the regularity hypothesis the polynomial $D_{\alpha, \alpha^{\prime}}^{2} f(x)+\beta$ totally splits in $\mathbb{F}_{q}[x]$.

- In Section 6, we show that we can choose a finite set of couples $\left(\alpha_{i}, \alpha_{i}^{\prime}\right)$ such that most polynomials $f \in \mathbb{F}_{q}[x]$ of degree $m$ satisfy the above regularity condition (Proposition 6.1). 
- Finally, Section 7 is devoted to the statement and the proof of the main theorem (Theorem 7.1).

To fix notation, throughout the whole paper we consider $n$ a non-negative integer and $q=2^{n}$. We denote by $\mathbb{F}_{q}$ the finite field with $q$ elements, by $\mathbb{F}_{q}[x]$ the ring of polynomials in one variable over $\mathbb{F}_{q}$ and by $\mathbb{F}_{q}[x]_{m}$ the $\mathbb{F}_{q}$-vector space of polynomials of $\mathbb{F}_{q}[x]$ of degree at most $m$. We will often consider a polynomial $f \in \mathbb{F}_{q}[x]$ of degree $m$, an element $\beta$ of $\mathbb{F}_{q}$ and distincts elements $\alpha$ and $\alpha^{\prime}$ in $\mathbb{F}_{q}^{*}$.

\section{The associated polynomial $L_{\alpha, \alpha^{\prime}}(f)$}

The derivative of a polynomial $f \in \mathbb{F}_{q}[x]$ along $\alpha \in \mathbb{F}_{q}^{*}$ is defined by

$$
D_{\alpha} f(x)=f(x)+f(x+\alpha)
$$

and its second derivative along $\left(\alpha, \alpha^{\prime}\right) \in \mathbb{F}_{q}^{2}$ is defined by

$D_{\alpha, \alpha^{\prime}}^{2} f(x)=D_{\alpha}\left(D_{\alpha^{\prime}} f\right)(x)=f(x)+f(x+\alpha)+f\left(x+\alpha^{\prime}\right)+f\left(x+\alpha+\alpha^{\prime}\right)$.

Actually $D_{\alpha, \alpha^{\prime}}^{2} f$ depends only on the $\mathbb{F}_{2}$-vector space generated by $\alpha$ and $\alpha^{\prime}$. If $f \in \mathbb{F}_{q}[x]$ is of odd degree $m$, then for any $\alpha \in \mathbb{F}_{q}^{*}$ the degree of $D_{\alpha} f$ is $m-1$. On the other hand, if $m$ is even then the degree of $D_{\alpha} f$ is less than or equal to $m-2$. Consequently, if $\alpha^{\prime} \in \mathbb{F}_{q}^{*}$ we obtain that the degree of $D_{\alpha, \alpha^{\prime}}^{2} f$ is less than or equal to $m-3$ when $m$ is odd, and less than or equal to $m-4$ otherwise. To any integer $m \geqslant 7$ we associate the following integer $d=d(m)$ (we will often omit the dependance in $m$ ).

Definition 2.1. Let $m$ be an integer greater or equal to 7 . If $m \equiv 0(\bmod 4)$ we set $d=\frac{m-4}{4}$, if $m \equiv 1(\bmod 4)$ we set $d=\frac{m-5}{4}$, if $m \equiv 2(\bmod 4)$ we set $d=\frac{m-6}{4}$ and if $m \equiv 3(\bmod 4)$ we set $d=\frac{m-3}{4}$.

We sum up the situation in the following table.

\begin{tabular}{|c|c|c|}
\hline$m(\bmod 4)$ & $\operatorname{deg} D_{\alpha, \alpha^{\prime}}^{2} f$ & $d$ \\
\hline 0 & $\leqslant m-4$ & $\frac{m-4}{4}$ \\
1 & $\leqslant m-3$ & $\frac{m-5}{4}$ \\
2 & $\leqslant m-4$ & $\frac{m-6}{4}$ \\
3 & $\leqslant m-3$ & $\frac{m-3}{4}$ \\
\hline
\end{tabular}

TABLE 1. Definition of $d$

Proposition 2.2. Let $\alpha, \alpha^{\prime} \in \mathbb{F}_{q}^{*}$ such that $\alpha \neq \alpha^{\prime}$ and let $f \in \mathbb{F}_{q}[x]$ be a polynomial of degree $m$. There exists a unique polynomial $g \in \mathbb{F}_{q}[x]$ of degree less than or equal to d such that

$$
D_{\alpha, \alpha^{\prime}}^{2} f(x)=g\left(x(x+\alpha)\left(x+\alpha^{\prime}\right)\left(x+\alpha+\alpha^{\prime}\right)\right) .
$$

Moreover, the map

$$
\begin{aligned}
L_{\alpha, \alpha^{\prime}}: \mathbb{F}_{q}[x] & \longrightarrow \mathbb{F}_{q}[x] \\
f & \longmapsto g
\end{aligned}
$$


is linear and $L_{\alpha, \alpha^{\prime}}\left(\mathbb{F}_{q}[x]_{m}\right)=\mathbb{F}_{q}[x]_{d}$.

Proof. Fix $f$ a polynomial of degree $m$ and $\alpha, \alpha^{\prime} \in \mathbb{F}_{q}^{*}$ such that $\alpha \neq \alpha^{\prime}$. Let us first prove the existence of $g$. If $D_{\alpha, \alpha^{\prime}}^{2} f$ is the zero polynomial then $g=0$ is suitable. Suppose now that $D_{\alpha, \alpha^{\prime}}^{2} f$ is non-zero and set $c$ for its leading coefficient and $\Lambda_{k}$ the set of its roots of multiplicity $k$ in an algebraic closure $\overline{\mathbb{F}}_{q}$ of $\mathbb{F}_{q}$. As $x \mapsto x+\alpha$ and $x \mapsto x+\alpha^{\prime}$ are two involutions of each set $\Lambda_{k}$, there exists $\Lambda_{k}^{\prime} \subset \Lambda_{k}$ such that:

$$
D_{\alpha, \alpha^{\prime}}^{2} f(x)=c \prod_{k \geqslant 1} \prod_{\lambda \in \Lambda_{k}^{\prime}}(x+\lambda)^{k}(x+\lambda+\alpha)^{k}\left(x+\lambda+\alpha^{\prime}\right)^{k}\left(x+\lambda+\alpha+\alpha^{\prime}\right)^{k} .
$$

Hence

$$
\begin{aligned}
D_{\alpha, \alpha^{\prime}}^{2} f(x) & =c \prod_{k \geqslant 1} \prod_{\lambda \in \Lambda_{k}^{\prime}}\left(x^{4}+\left(\alpha^{2}+\alpha^{\prime 2}+\alpha \alpha^{\prime}\right) x^{2}+\left(\alpha^{2} \alpha^{\prime}+\alpha \alpha^{\prime 2}\right) x+\lambda^{4}+\left(\alpha^{2}+\alpha^{\prime 2}+\alpha \alpha^{\prime}\right) \lambda^{2}+\left(\alpha^{2} \alpha^{\prime}+\alpha \alpha^{\prime 2}\right) \lambda\right)^{k} \\
& =c \prod_{k \geqslant 1} \prod_{\lambda \in \Lambda_{k}^{\prime}}\left(x(x+\alpha)\left(x+\alpha^{\prime}\right)\left(x+\alpha+\alpha^{\prime}\right)+\lambda(\lambda+\alpha)\left(\lambda+\alpha^{\prime}\right)\left(\lambda+\alpha+\alpha^{\prime}\right)\right)^{k} .
\end{aligned}
$$

Then the polynomial $g$ defined by

$$
g(x)=c \prod_{k \geqslant 1} \prod_{\lambda \in \Lambda_{k}^{\prime}}\left(x+\lambda(\lambda+\alpha)\left(\lambda+\alpha^{\prime}\right)\left(\lambda+\alpha+\alpha^{\prime}\right)\right)^{k}
$$

satisfies $g\left(x(x+\alpha)\left(x+\alpha^{\prime}\right)\left(x+\alpha+\alpha^{\prime}\right)\right)=D_{\alpha, \alpha^{\prime}}^{2} f(x)$ and has degree at most $d$. To prove that $g \in \mathbb{F}_{q}[x]$, one can quote linear algebra arguments. Actually, solving $g\left(x(x+\alpha)\left(x+\alpha^{\prime}\right)\left(x+\alpha+\alpha^{\prime}\right)\right)=D_{\alpha, \alpha^{\prime}}^{2} f(x)$ amounts to solving an affine equation with coefficients in $\mathbb{F}_{q}$ and we have already proven that this equation admits solutions with coefficients in $\overline{\mathbb{F}}_{q}$. As the existence of solutions of such affine equations does not depend of the extension field considered, we have solutions with coefficients in $\mathbb{F}_{q}$. The uniqueness is a consequence of the linearity of composition. To prove the surjectivity of $L_{\alpha, \alpha^{\prime}}$, we will determine the dimension of its kernel and apply the rank-nullity theorem. Note that $f \in \operatorname{Ker} L_{\alpha, \alpha^{\prime}}$ if and only if $D_{\alpha, \alpha^{\prime}}^{2} f=0$. But $D_{\alpha, \alpha^{\prime}}^{2} f=$ $D_{\alpha} D_{\alpha^{\prime}} f$, so $\operatorname{Ker} L_{\alpha, \alpha^{\prime}}=D_{\alpha^{\prime}}^{-1}\left(\operatorname{Ker} D_{\alpha}\right)$. Classical linear algebra properties give the equality $\operatorname{dim} \operatorname{Ker} L_{\alpha, \alpha^{\prime}}=\operatorname{dim}\left(\operatorname{Im} D_{\alpha^{\prime}} \cap \operatorname{Ker} D_{\alpha}\right)+\operatorname{dim} \operatorname{Ker}\left(D_{\alpha^{\prime}}\right)$. We conclude separating cases according to the congruence of $m$ modulo 4 and using Lemma 2.3 .

For simplicity of notation we continue to write $D_{\alpha}$ for the restriction of $D_{\alpha}$ to the subspace of polynomials of degree less than or equal to $m$. We also use the notations $\lfloor a\rfloor$ for the greatest integer less than or equal to $a$ and $\lceil a\rceil$ for the least integer greater than or equal to $a$.

Lemma 2.3. Let $\alpha$ and $\alpha^{\prime}$ be two distinct elements in $\mathbb{F}_{q}^{*}$. We have:

(i) $\operatorname{Ker} D_{\alpha}=\{h(x(x+\alpha)) \mid \operatorname{deg}(h) \leqslant\lfloor m / 2\rfloor\}$. 
(ii) $\operatorname{Im} D_{\alpha}=\{h(x(x+\alpha)) \mid \operatorname{deg}(h) \leqslant\lceil m / 2\rceil-1\}$.

(iii) If $m$ is odd, then

$\operatorname{Im} D_{\alpha^{\prime}} \cap \operatorname{Ker} D_{\alpha}=\left\{h\left(x(x+\alpha)\left(x+\alpha^{\prime}\right)\left(x+\alpha+\alpha^{\prime}\right)\right) \mid \operatorname{deg}(h) \leqslant m / 4\right\}$.

(iv) If $m$ is even, then

$\operatorname{Im} D_{\alpha^{\prime}} \cap \operatorname{Ker} D_{\alpha}=\left\{h\left(x(x+\alpha)\left(x+\alpha^{\prime}\right)\left(x+\alpha+\alpha^{\prime}\right)\right) \mid \operatorname{deg}(h) \leqslant(m-2) / 4\right\}$.

Proof. If $D_{\alpha} f=0$ then $f(x)=f(x+\alpha)$. The map $x \mapsto x+\alpha$ induces a bijection onto the sets of the roots of $f$ of same multiplicity. Using the method of the proof of Proposition 2.2 we prove (i). We deduce (ii) proving an easy inclusion and the rank-nullity theorem. To prove (iii), use that if $m$ is odd then $\operatorname{Im} D_{\alpha^{\prime}}=\operatorname{Ker} D_{\alpha^{\prime}}$ by (i) and (ii). Suppose that $f \in$ $\operatorname{Ker} D_{\alpha^{\prime}} \cap \operatorname{Ker} D_{\alpha}$. If $x_{0}$ is a root of $f$ of multiplicity $k$, so are $x_{0}+\alpha, x_{0}+\alpha^{\prime}$ and $x_{0}+\alpha+\alpha^{\prime}$, and we can use the method of the proof of Proposition 2.2 . We prove (iv) using the same method and noticing that the intersection $\operatorname{Im} D_{\alpha^{\prime}} \cap \operatorname{Ker} D_{\alpha}$ consists of the polynomials of $\operatorname{Ker} D_{\alpha^{\prime}} \cap \operatorname{Ker} D_{\alpha}$ of degree less than or equal to $m-2$.

\section{Monodromy groups And Morse polynomials}

Let $g \in \mathbb{F}_{q}[x]$ be a polynomial of degree $d$. We consider the field extension $\mathbb{F}_{q}(u) / \mathbb{F}_{q}(t)$ corresponding to the polynomial $g$ where $t$ is transcendental over $\mathbb{F}_{q}$ i.e. with $u$ such that $g(u)-t=0$. Denote by $F$ the Galois closure of $\mathbb{F}_{q}(u) / \mathbb{F}_{q}(t)$, i.e. $F$ is the splitting field of $g(x)-t$ over $\mathbb{F}_{q}(t)$. The Galois group $\operatorname{Gal}\left(F / \mathbb{F}_{q}(t)\right)$ is called the arithmetic monodromy group of $g$. Let $\mathbb{F}_{q}^{F}$ be the algebraic closure of $\mathbb{F}_{q}$ in $F$. Then the Galois group $\operatorname{Gal}\left(F / \mathbb{F}_{q}^{F}(t)\right)$ is a normal subgroup of $\operatorname{Gal}\left(F / \mathbb{F}_{q}(t)\right)$ called the geometric monodromy group of $g$.

The polynomial $g$ is said to be Morse (see [9] p. 39) if $g$, viewed as a ramified covering $g: \mathbb{P}^{1} \longrightarrow \mathbb{P}^{1}$ of degree $d$, is such that above each affine branch point there is only one ramification point and the ramification index of such points is 2 . In even characteristic, this notion has to be precised: following Geyer in the Appendix of [4], the polynomial $g$ is said to be Morse if the three following conditions hold:

a) $g^{\prime}(\tau)=0$ implies that $g^{[2]}(\tau)=0$ where $g^{[2]}$ is the second HasseSchmidt derivative,

b) $g^{\prime}(\tau)=g^{\prime}(\eta)=0$ and $g(\tau)=g(\eta)$ imply $\tau=\eta$,

c) the degree of $g$ is not divisible by the characteristic of $\mathbb{F}_{q}$.

For Morse polynomials $g$, the general form of the Hilbert theorem given by Serre in Theorem 4.4.5 of [9] adapted to the even characteristic in Proposition 4.2. in the Appendix by Geyer of [4] implies that the geometric monodromy group $\operatorname{Gal}\left(F / \mathbb{F}_{q}^{F}(t)\right)$ is the symmetric group $\mathfrak{S}_{d}$. Moreover, it is a subgroup of the arithmetic monodromy group $\operatorname{Gal}\left(F / \mathbb{F}_{q}(t)\right)$ and this last group is also contained in $\mathfrak{S}_{d}$, hence they coincide. 
Now let us return to our situation. Let $\alpha, \alpha^{\prime}$ be two distincts elements of $\mathbb{F}_{q}^{*}$. Let $m$ be an integer and $d=d(m)$ defined in Table 1 , Let $f \in \mathbb{F}_{q}[x]$ be a polynomial of degree $m$. Let us consider the polynomial $g:=L_{\alpha, \alpha^{\prime}}(f) \in$ $\mathbb{F}_{q}[x]$ of degree $\leqslant d$ such that

$$
g\left(x(x+\alpha)\left(x+\alpha^{\prime}\right)\left(x+\alpha+\alpha^{\prime}\right)\right)=D_{\alpha, \alpha^{\prime}}^{2} f(x)
$$

whose existence follows from Proposition 2.2.

Proposition 3.1. If $f$ is a polynomial of degree $m$ such that the polynomial $L_{\alpha, \alpha^{\prime}}(f)$ is of degree exactly $d$ and is Morse then the geometric monodromy group, and then also the arithmetic monodromy group of the polynomial $L_{\alpha, \alpha^{\prime}}(f)$ is the symmetric group $\mathfrak{S}_{d}$. Hence the extension $F / \mathbb{F}_{q}(t)$ is regular i.e. $\mathbb{F}_{q}^{F}=\mathbb{F}_{q}$.

Proof. By the previous paragraph we have that the geometric and the arithmetic monodromy groups coincide, which gives the regularity property.

Note that if $L_{\alpha, \alpha^{\prime}}(f)$ is of degree exactly $d$ and is Morse then Condition (c) says that $d$ must be odd. This is equivalent to say that $m \equiv 0,1,2$ or 7 $(\bmod 8)$.

Now we give a lower bound for the number of polynomials $f$ such that $L_{\alpha, \alpha^{\prime}}(f)$ is Morse.

Proposition 3.2. Let $m \geqslant 7$ such that $m \equiv 0,1,2$ or $7(\bmod 8)$ and $d$ as defined in Definition 2.1. There exists an integer $\tilde{d}>0$ depending only on $d$ such that for any couple $\left(\alpha, \alpha^{\prime}\right)$ of distinct elements of $\mathbb{F}_{q}^{*}$ the number of polynomials $f$ of $\mathbb{F}_{q}[x]$ of degree at most $m$ such that $L_{\alpha, \alpha^{\prime}}(f)$ is non-Morse is bounded by $\tilde{d} q^{m}$.

Proof. The loci of non-Morse polynomials $g=\sum_{j=0}^{d} b_{d-j} x^{j}$ of $\mathbb{F}_{q}[x]$ of degree $d$ is a Zariski-closed subset of the $(d+1)$-dimensional affine space with coordinates $b_{0}, \ldots, b_{d}$ given by Geyer in Proposition 4.3 of the Appendix of [4]. Indeed, the above condition (a) means that $g^{\prime}$ and $g^{[2]}$ have no common root, i.e. the resultant $R\left(b_{0}, \ldots, b_{d}\right)$ of the polynomials $g^{\prime}$ and $g^{[2]}$ is nonzero. Condition (b) above means that the product

$$
\Pi=\prod_{i \neq j}\left(g\left(\eta_{i}\right)-g\left(\eta_{j}\right)\right)
$$

where $\eta_{i}$ are the roots of $g$ does not vanish. By the theorem on symmetric functions, $\Pi=\Pi\left(b_{0}, \ldots, b_{d}\right)$ is a polynomial in the coefficients of $g$.

Finally the polynomials $f=\sum_{j=0}^{m} a_{j} x^{m-j}$ such that $L_{\alpha, \alpha^{\prime}}(f)$ is nonMorse are those such that

$$
R \circ L_{\alpha, \alpha^{\prime}}\left(a_{0}, \ldots, a_{m}\right)=0 \text { or } \Pi \circ L_{\alpha, \alpha^{\prime}}\left(a_{0}, \ldots, a_{m}\right)=0 .
$$

The polynomials $R$ and $\Pi$ are proven to be non-zero in Geyer's Appendix. By Proposition 2.2 we know that $L_{\alpha, \alpha^{\prime}}$ is surjective. Hence $R \circ L_{\alpha, \alpha^{\prime}}$ and $\Pi \circ L_{\alpha, \alpha^{\prime}}$ are non-zero, and then define hypersurfaces in $\mathbb{A}^{m+1}\left(\overline{\mathbb{F}}_{q}\right)$. Their 
numbers of rational points are bounded respectively by $C_{R} q^{m}$ and $C_{\Pi} q^{m}$ where $C_{R}$ and $C_{\Pi}$ are respectively the degree of $R \circ L_{\alpha, \alpha^{\prime}}$ and $\Pi \circ L_{\alpha, \alpha^{\prime}}$ (see for example Section 5 of Chapter 1 in [1]). Since $L_{\alpha, \alpha^{\prime}}$ is linear, one can bound $C_{R}$ and $C_{\Pi}$ by the degree $d_{R}$ of $R$ and the degree $d_{\Pi}$ of $\Pi$ and then one can bound $C_{R}+C_{\Pi}$ by $\tilde{d}=d_{R}+d_{\Pi}$, which does not depend on the choice of $\left(\alpha, \alpha^{\prime}\right)$.

\section{Geometric and arithmetic monodromy groups of $D_{\alpha, \alpha^{\prime}}^{2} f$}

In the whole section we consider a polynomial $f$ of degree $m$ with $m \equiv$ $0,1,2$ or $7(\bmod 8)$ and two distincts elements $\alpha, \alpha^{\prime}$ of $\mathbb{F}_{q}^{*}$ such that the polynomial $g:=L_{\alpha, \alpha^{\prime}}(f)$ is of degree exactly $d$ (given by Table 11) and is Morse. We denote by $u_{0}, \ldots, u_{d-1}$ the roots of $L_{\alpha, \alpha^{\prime}}(f)(u)+t$, and for $i=0, \ldots, d-1$ we denote by $x_{i}$ a solution of the equation

$$
x(x+\alpha)\left(x+\alpha^{\prime}\right)\left(x+\alpha+\alpha^{\prime}\right)=u_{i} .
$$

Hence $D_{\alpha, \alpha^{\prime}}^{2} f\left(x_{i}\right)=t$. For convenience, we will note

$$
S_{\gamma}(X)=X(X+\gamma)
$$

for $\gamma \in \mathbb{F}_{q}$ and

$$
T_{\gamma_{1}, \gamma_{2}}(X)=X\left(X+\gamma_{1}\right)\left(X+\gamma_{2}\right)\left(X+\gamma_{1}+\gamma_{2}\right)
$$

for $\left(\gamma_{1}, \gamma_{2}\right) \in \mathbb{F}_{q}^{2}$. We will use the following equalities (easy to check):

$$
S_{\gamma_{1} \gamma_{2}}\left(x_{i}\left(x_{i}+\gamma_{3}\right)\right)=u_{i} \text { and } S_{\gamma_{1} \gamma_{2} \gamma_{3}}\left(\gamma_{3} x_{i}\left(x_{i}+\gamma_{3}\right)\right)=\gamma_{3}^{2} u_{i}
$$

where $\left\{\gamma_{1}, \gamma_{2}, \gamma_{3}\right\}=\left\{\alpha, \alpha^{\prime}, \alpha+\alpha^{\prime}\right\}$.

We consider, for $i \in\{0, \ldots, d-1\}$, the extensions $F\left(x_{i}\right) / F$ and $\Omega$ their compositum (where the field $F$ is defined in the previous section). Then $\Omega$ is the splitting field of $D_{\alpha, \alpha^{\prime}}^{2} f(x)+t$ and $\operatorname{Gal}\left(\Omega / \mathbb{F}_{q}(t)\right)$ is the arithmetic monodromy group of $D_{\alpha, \alpha^{\prime}}^{2} f$ whereas $\operatorname{Gal}\left(\Omega / \mathbb{F}_{q}^{\Omega}(t)\right)$ is the geometric monodromy group of $D_{\alpha, \alpha^{\prime}}^{2} f$, where we denote by $\mathbb{F}_{q}^{\Omega}$ the algebraic closure of $\mathbb{F}_{q}$ in $\Omega$. The figure below sums up the situation whose details will be explained in this section. 


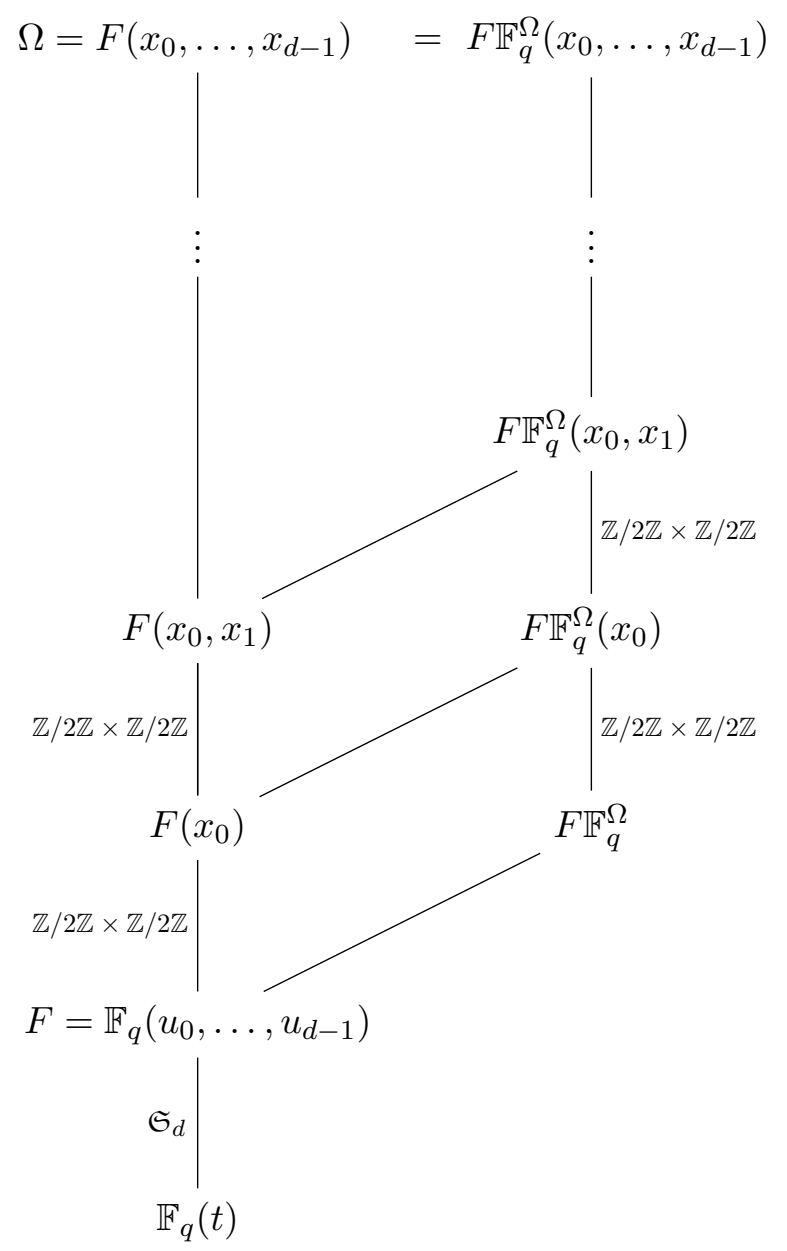

The following lemma gives conditions for two Artin-Schreier extensions to be equal.

Lemma 4.1. Let $k\left(y_{1}\right)$ and $k\left(y_{2}\right)$ be two Artin-Schreier extensions of a field $k$ of characteristic 2. Suppose that $y_{i}^{2}+\gamma_{i} y_{i}=w_{i}$ for $i \in\{1,2\}$ with $\gamma_{i}$ and $w_{i}$ in $k^{*}$. Then $k\left(y_{1}\right)=k\left(y_{2}\right)$ if and only if $\gamma_{2} y_{1}+\gamma_{1} y_{2} \in k$.

Proof. Suppose that $k\left(y_{1}\right)=k\left(y_{2}\right)$. Consequently $y_{2} \in k\left(y_{1}\right)$ and there exists $(a, b) \in k^{2}$ such that $y_{2}=a+b y_{1}$. Consider the element $\tau$ of $\mathrm{Gal}\left(k\left(y_{1}\right) / k\right)$ distinct from the identity. It maps $y_{1}$ to $y_{1}+\gamma_{1}$. We have $\tau\left(y_{2}\right)=a+b y_{1}+b \gamma_{1}$ i.e. $\tau\left(y_{2}\right)=y_{2}+b \gamma_{1}$. But $\tau\left(y_{2}\right)$ is a root of $y^{2}+\gamma_{2} y=w_{2}$, so $\tau\left(y_{2}\right)=y_{2}$ or $\tau\left(y_{2}\right)=y_{2}+\gamma_{2}$. In the first case $\tau$ would be the identity, a contradiction. Hence $\tau\left(y_{2}\right)=y_{2}+\gamma_{2}$ and then $y_{2}+\gamma_{2}=y_{2}+b \gamma_{1}$, which implies that $\gamma_{2}=b \gamma_{1}$. So we get $\gamma_{2} y_{1}+\gamma_{1} y_{2}=b \gamma_{1} y_{1}+\gamma_{1} y_{2}=$ $b \gamma_{1} y_{1}+a \gamma_{1}+b \gamma_{1} y_{1}=a \gamma_{1} \in k$ where we used that $y_{2}=a+b y_{1}$. The converse is straightforward. 
Now we prove that a linear combination of the roots $u_{j}$ with no pole actually involves all of them.

Lemma 4.2. Let $\kappa$ be $\mathbb{F}_{q}$ or $\mathbb{F}_{q}^{\Omega}$. For each place $\wp$ of $\kappa\left(u_{0}, \ldots, u_{d-1}\right)$ above the place $\infty$ of $\kappa(t)$ and each $j \in\{0, \ldots, d-1\}$ we have that $u_{j}$ has a simple pole at $\wp$. Moreover, let $J \subset\{0, \ldots, d-1\}$ and let $c_{0}, \ldots, c_{d-1} \in \mathbb{F}_{q}^{*}$. If $J$ is neither empty nor the whole set then $\sum_{j \in J} c_{j} u_{j}$ has a pole at a place of $\kappa\left(u_{0}, \ldots, u_{d-1}\right)$ lying over the infinite place $\infty$ of $\kappa(t)$.

Proof. Fix $\wp$ a place above $\infty$ and $u_{i}$ a root of $g(u)-t$. We have $v_{\wp}\left(g\left(u_{i}\right)\right)=$ $v_{\wp}(t)$ and $v_{\wp}(t)=e(\wp \mid \infty) v_{\infty}(t)$ where $e(\wp \mid \infty)$ is the ramification index of $\wp$ over $\infty$. By 9], p. 41, we have that the inertia group at infinity is generated by a $d$-cycle, so we have $e(\wp \mid \infty)=d$ and then $v_{\wp}(t)=-d$. Now $v_{\wp}\left(g\left(u_{i}\right)\right)=v_{\wp}\left(b_{0} u_{i}^{d}+b_{1} u_{i}^{d-1}+\cdots+b_{d}\right)$ so using the properties of the valuation of a sum we deduce that $v_{\wp}\left(u_{i}\right)=-1$.

The proof of the second part of the lemma is inspired by [11. To obtain a contradiction, suppose that $J \subset\{0, \ldots, d-1\}$ and that $j_{0} \in J$ whereas $j_{1} \in\{0, \ldots, d-1\} \backslash J$. Suppose also that $\sum_{j \in J} c_{j} u_{j}$ has no pole in places above $\infty$. Then it has no pole at all, and so it is constant, i.e. it belongs to $\kappa$. By Proposition 3.1 we have that $\operatorname{Gal}\left(\kappa\left(u_{0}, \ldots, u_{d-1}\right) / \kappa(t)\right)$ is $\mathfrak{S}_{d}$. Let us choose the automorphism $\theta$ corresponding to the transposition $\left(j_{0} j_{1}\right)$ and let us apply $\theta$ to $\sum_{j \in J} c_{j} u_{j}$. We obtain $\sum_{j \in J \backslash j_{0}} c_{j} u_{j}+c_{j_{0}} u_{j_{0}}=\sum_{j \in J \backslash j_{0}} c_{j} u_{j}+$ $c_{j_{0}} u_{j_{1}}$. We deduce $u_{j_{0}}=u_{j_{1}}$, a contradiction.

The following lemma, used with Lemma 4.1, will enable us to distinguish different Artin-Schreier subextensions of $\Omega$.

Lemma 4.3. Let $\widetilde{F}$ be $F$ or $F \mathbb{F}_{q}^{\Omega}$. Let $J$ be a non-empty strict subset of $\{0, \ldots, d-1\}$ and for all $j \in J$ consider any $\gamma_{j} \in\left\{\alpha, \alpha^{\prime}, \alpha+\alpha^{\prime}\right\}$. Then

$$
\sum_{j \in J} \gamma_{j} x_{j}\left(x_{j}+\gamma_{j}\right) \notin \widetilde{F} \text {. }
$$

Proof. In order to obtain a contradiction suppose that $\sum_{j \in J} \gamma_{j} x_{j}\left(x_{j}+\gamma_{j}\right) \in$ $\widetilde{F}$. Lemma 4.2 implies that $\sum_{j \in J} \gamma_{j}^{2} u_{j}$ has a pole at a place $\wp$ of $\widetilde{F}$ above $\infty$. Moreover this pole is simple as for all $j \in\{1, \ldots, d-1\}$ the root $u_{j}$ has a simple pole by Lemma 4.2. Now consider $A=\sum_{j \in J} \gamma_{j} x_{j}\left(x_{j}+\gamma_{j}\right)$ and $B=\sum_{j \in J} \gamma_{j} x_{j}\left(x_{j}+\gamma_{j}\right)+\alpha \alpha^{\prime}\left(\alpha+\alpha^{\prime}\right)$. If $A$ (and thus $B$ ) belongs to $\widetilde{F}$, one can consider the valuation of $A$ and $B$ at $\wp$. As

$$
A . B=S_{\alpha \alpha^{\prime}\left(\alpha+\alpha^{\prime}\right)}\left(\sum_{j \in J} \gamma_{j} x_{j}\left(x_{j}+\gamma_{j}\right)\right)=\sum_{j \in J} \gamma_{j}^{2} u_{j},
$$

it follows that either $A$ or $B$ has a pole. Since $A$ and $B$ differ by a constant, it follows that both of them have a pole and the order of multiplicity is the same. Thus we obtain $2 v_{\wp}(A)=-1$ which is a contradiction. 
The following lemma establishes the base case of the induction proof of Proposition 4.5.

Lemma 4.4. Let $\widetilde{F}$ be $F$ or $F \mathbb{F}_{q}^{\Omega}$. Let $i \in\{0, \ldots, d-1\}$. The field $\widetilde{F}\left(x_{i}\right)$ is a degree 4 extension of $\widetilde{F}$ and its Galois group is $\mathbb{Z} / 2 \mathbb{Z} \times \mathbb{Z} / 2 \mathbb{Z}$. The three subextensions of degree 2 are the subextensions $\widetilde{F}\left(x_{i}\left(x_{i}+\gamma\right)\right)$ where $\gamma \in\left\{\alpha, \alpha^{\prime}, \alpha+\alpha^{\prime}\right\}$. The following diagram sums up the situation:

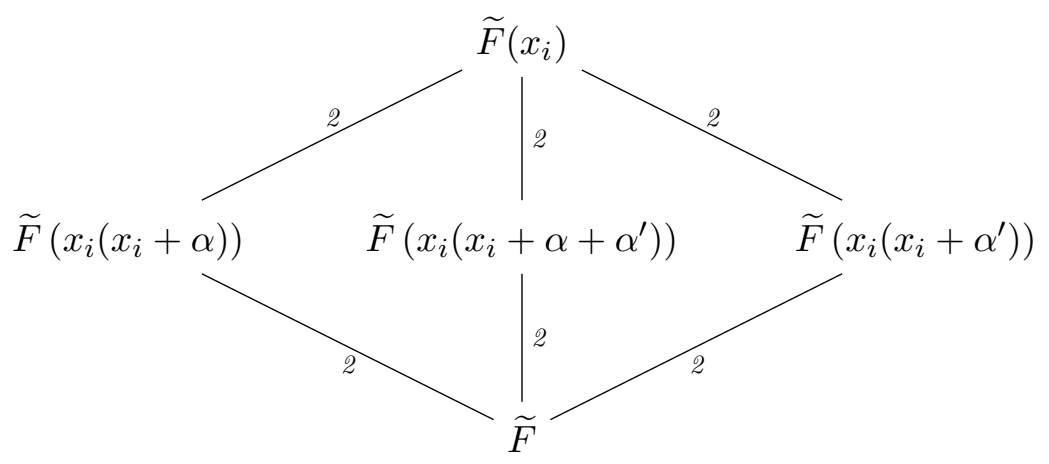

Proof. First notice that $x_{i} \notin \widetilde{F}$. Otherwise, one would obtain a contradiction considering the equality $x_{i}\left(x_{i}+\alpha\right)\left(x_{i}+\alpha^{\prime}\right)\left(x_{i}+\alpha+\alpha^{\prime}\right)=u_{i}$, the valuation of $x_{i}$ at a place above $\infty$, and the valuation of $u_{i}$ at this place which is -1 . Now suppose that $\left[\widetilde{F}\left(x_{i}\right): \widetilde{F}\right]=2$. We would have a degree 2 factor of the polynomial $X(X+\alpha)\left(X+\alpha^{\prime}\right)\left(X+\alpha+\alpha^{\prime}\right)+u_{i}$ and then an element $x_{i}\left(x_{i}+\gamma\right)$ with $\gamma \in\left\{\alpha, \alpha^{\prime}, \alpha+\alpha^{\prime}\right\}$ would be in $\widetilde{F}$, contradicting Lemma 4.3, So $\left[\widetilde{F}\left(x_{i}\right)\right.$ : $\widetilde{F}]=4$, and $T_{\alpha, \alpha^{\prime}}(X)+u_{i}$ is the minimal polynomial of $x_{i}$ over $\widetilde{F}$. It enables us to define, for any $\gamma \in\left\{\alpha, \alpha^{\prime}, \alpha+\alpha^{\prime}\right\}$, an element $\tau_{\gamma}$ of Gal $\left(\widetilde{F}\left(x_{i}\right) / \widetilde{F}\right)$ by $\tau_{\gamma}\left(x_{i}\right)=x_{i}+\gamma$. We thus have Gal $\left(\widetilde{F}\left(x_{i}\right) / \widetilde{F}\right)=\left\{i d, \tau_{\alpha}, \tau_{\alpha^{\prime}}, \tau_{\alpha+\alpha^{\prime}}\right\}$ and thus $\operatorname{Gal}\left(\widetilde{F}\left(x_{i}\right) / \widetilde{F}\right) \simeq \mathbb{Z} / 2 \mathbb{Z} \times \mathbb{Z} / 2 \mathbb{Z}$. There are three subextensions of degree 2 , namely the subextensions $\widetilde{F}\left(x_{i}\left(x_{i}+\gamma\right)\right)$ where $\gamma \in\left\{\alpha, \alpha^{\prime}, \alpha+\alpha^{\prime}\right\}$. Their stabilizers are respectively the index 2 subgroups $\left\{\mathrm{id}, \tau_{\gamma}\right\}$.

The previous lemmas enable us to determine in the following two propositions the Galois groups of $\widetilde{F}\left(x_{0}, \ldots, x_{d-2}\right)$ and $\Omega=\widetilde{F}\left(x_{0}, \ldots, x_{d-1}\right)$ over $\widetilde{F}$ where $\widetilde{F}$ is equal to $F$ or $F \mathbb{F}_{q}^{\Omega}$.

Proposition 4.5. Let $\tilde{F}$ be $F$ or $F \mathbb{F}_{q}^{\Omega}$ and let $r$ be an integer such that $0 \leqslant r \leqslant d-2$. Then:

(i) The field $\widetilde{F}\left(x_{0}, \ldots, x_{r}\right)$ is an extension of degree $4^{r+1}$ of $\widetilde{F}$.

(ii) The Galois group $\operatorname{Gal}\left(\widetilde{F}\left(x_{0}, \ldots, x_{r}\right) / \widetilde{F}\right)$ is $(\mathbb{Z} / 2 \mathbb{Z} \times \mathbb{Z} / 2 \mathbb{Z})^{r+1}$. It is generated by the automorphisms $\tau_{i, \gamma}$ for $i \in\{0, \ldots, r\}$ and $\gamma \in\left\{\alpha, \alpha^{\prime}, \alpha+\alpha^{\prime}\right\}$ (where $\tau_{i, \gamma}$ maps $x_{i}$ to $x_{i}+\gamma$ and leaves $x_{j}$ invariant for $j \neq i$ ). 
(iii) There are $4^{r+1}-1$ quadratic extensions of $\widetilde{F}$ contained in $\widetilde{F}\left(x_{0}, \ldots, x_{r}\right)$. These extensions are the fields $\widetilde{F}\left(\sum_{j \in J} \gamma_{j} x_{j}\left(x_{j}+\gamma_{j}\right)\right)$ with non-empty $J \subset$ $\{0, \ldots, r\}$ and $\gamma_{j} \in\left\{\alpha, \alpha^{\prime}, \alpha+\alpha^{\prime}\right\}$ for all $j \in J$.

Proof. We proceed by induction. The case $r=0$ follows from Lemma 4.4. Assuming that the proposition holds for $r-1$, with $0<r \leqslant d-$ 2 , we will prove it for $r$. We consider the extension $\widetilde{F}\left(x_{0}, \ldots, x_{r-1}\right)\left(x_{r}\right)$ of $\widetilde{F}\left(x_{0}, \ldots, x_{r-1}\right)$. We first prove that the degree of this extension is 4 and that the minimal polynomial of $x_{r}$ is $T_{\alpha, \alpha^{\prime}}(X)+u_{r}$. Suppose it is false: either $x_{r} \in \widetilde{F}\left(x_{0}, \ldots, x_{r-1}\right)$ or $T_{\alpha, \alpha^{\prime}}(X)+u_{r}$ (which is equal to $\left.\left(x+x_{r}\right)\left(x+x_{r}+\alpha\right)\left(x+x_{r}+\alpha^{\prime}\right)\left(x+x_{r}+\alpha+\alpha^{\prime}\right)\right)$ has a degree 2 factor in $\widetilde{F}\left(x_{0}, \ldots, x_{r-1}\right)[X]$, hence there exists $\gamma \in\left\{\alpha, \alpha^{\prime}, \alpha+\alpha^{\prime}\right\}$ such that $x_{r}\left(x_{r}+\gamma\right) \in \widetilde{F}\left(x_{0}, \ldots, x_{r-1}\right)$. In both cases we would have an extension $\widetilde{F}\left(x_{r}\left(x_{r}+\gamma\right)\right)$ of degree 2 of $\widetilde{F}$ contained in $\widetilde{F}\left(x_{0}, \ldots, x_{r-1}\right)$. Use the induction hypothesis: it is one of the subextensions $\widetilde{F}\left(\sum_{j \in J} \gamma_{j} x_{j}\left(x_{j}+\gamma_{j}\right)\right)$ with a non-empty subset $J \subset\{0, \ldots, r-1\}$. By Lemma 4.1 and identities (11) it follows that $\sum_{j \in J} \gamma_{j} x_{j}\left(x_{j}+\gamma_{j}\right)+\gamma x_{r}\left(x_{r}+\gamma\right) \in \widetilde{F}$, a contradiction with Lemma 4.3. We conclude that the extension $\widetilde{F}\left(x_{0}, \ldots, x_{r}\right) / \widetilde{F}\left(x_{0}, \ldots, x_{r-1}\right)$ has degree 4 and then $\widetilde{F}\left(x_{0}, \ldots, x_{r}\right) / \widetilde{F}$ has degree $4^{r+1}$.

But we can define $4^{r+1}$ different $\widetilde{F}$-automorphisms of $\widetilde{F}\left(x_{0}, \ldots, x_{r}\right)$ by sending for any $i \in\{0, \ldots, r\}$ the element $x_{i}$ to $x_{i}+\gamma_{i}$ with $\gamma_{i} \in\left\{0, \alpha, \alpha^{\prime}, \alpha+\right.$ $\left.\alpha^{\prime}\right\}$. Since all these automorphisms (apart from the identity) have order 2 , the Galois group Gal $\left(\widetilde{F}\left(x_{0}, \ldots, x_{r}\right) / \widetilde{F}\right)$ is isomorphic to $(\mathbb{Z} / 2 \mathbb{Z} \times \mathbb{Z} / 2 \mathbb{Z})^{r+1}$.

For any non-empty subset $J \subset\{0, \ldots, r\}$ and for any choice of a family $\left(\gamma_{j}\right)_{j \in J}$ of elements of $\left\{\alpha, \alpha^{\prime}, \alpha+\alpha^{\prime}\right\}$, we know that $\sum_{j \in J} \gamma_{j} x_{j}\left(x_{j}+\gamma_{j}\right)$ is a root of $S_{\alpha \alpha^{\prime}\left(\alpha+\alpha^{\prime}\right)}(X)+\sum_{j \in J} \gamma_{j}^{2} u_{j}$. By Lemma 4.3 we also know that this sum does not belong to $\widetilde{F}$, so the extensions $\widetilde{F}\left(\sum_{j \in J} \gamma_{j} x_{j}\left(x_{j}+\gamma_{j}\right)\right)$ are quadratic.

We claim that we obtain this way $4^{r+1}-1$ different quadratic extensions between $\widetilde{F}$ and $\widetilde{F}\left(x_{0}, \ldots, x_{r}\right)$. To prove our claim, we consider two families $\left(\gamma_{j}\right)_{j \in J}$ and $\left(\gamma_{j}^{\prime}\right)_{j \in J^{\prime}}$ of elements of $\left\{\alpha, \alpha^{\prime}, \alpha+\alpha^{\prime}\right\}$ where $J$ and $J^{\prime}$ are two subsets of $\{0, \ldots, r\}$. We notice that if $j \in J \cap J^{\prime}$ is such that $\gamma_{j} \neq \gamma_{j}^{\prime}$ then $\gamma_{j} x_{j}\left(x_{j}+\gamma_{j}\right)+\gamma_{j}^{\prime} x_{j}\left(x_{j}+\gamma_{j}^{\prime}\right)=\gamma_{j}^{\prime \prime} x_{j}\left(x_{j}+\gamma_{j}^{\prime \prime}\right)$ where $\left\{\gamma_{j}, \gamma_{j}^{\prime}, \gamma_{j}^{\prime \prime}\right\}=$ $\left\{\alpha, \alpha^{\prime}, \alpha+\alpha^{\prime}\right\}$. Then if $\widetilde{F}\left(\sum_{j \in J} \gamma_{j} x_{j}\left(x_{j}+\gamma_{j}\right)\right)=\widetilde{F}\left(\sum_{j \in J^{\prime}} \gamma_{j} x_{j}\left(x_{j}+\gamma_{j}\right)\right)$ we obtain by Lemma 4.1 a sum

$$
\sum_{j \in J \backslash J^{\prime}} \gamma_{j} x_{j}\left(x_{j}+\gamma_{j}\right)+\sum_{j \in J^{\prime} \backslash J} \gamma_{j}^{\prime} x_{j}\left(x_{j}+\gamma_{j}^{\prime}\right)+\sum_{\substack{j \in J \cap J^{\prime} \\ \gamma_{j} \neq \gamma_{j}^{\prime}}} \gamma_{j}^{\prime \prime} x_{j}\left(x_{j}+\gamma_{j}^{\prime \prime}\right)
$$

which is in $\widetilde{F}$. By Lemma 4.3, it implies $J=J^{\prime}$ and $\gamma_{j}=\gamma_{j}^{\prime}$ for all $j \in J$. 
Finally, we claim that these $4^{r+1}-1$ quadratic extensions are the only ones. Indeed, the quadratic extensions are in correspondence with the subgroups of $(\mathbb{Z} / 2 \mathbb{Z} \times \mathbb{Z} / 2 \mathbb{Z})^{r+1}$ of index 2 . These subgroups are the hyperplanes of $(\mathbb{Z} / 2 \mathbb{Z})^{2 r+2}$ and there are $4^{r+1}-1$ such hyperplanes.

Recall that in this section the polynomial $g=L_{\alpha, \alpha^{\prime}}(f)=\sum_{i=0}^{d} b_{d-i} x^{i}$ is supposed to be Morse and to have degree exactly $d$. We can now establish the main result of this section: we give a sufficient condition on $b_{1} / b_{0}$ for $\Omega / \mathbb{F}_{q}(t)$ to be regular, which is a necessary condition to apply the Chebotarev theorem.

Proposition 4.6. If there exists $x \in \mathbb{F}_{q}$ such that

$$
\frac{b_{1}}{b_{0}}=x(x+\alpha)\left(x+\alpha^{\prime}\right)\left(x+\alpha+\alpha^{\prime}\right)
$$

then we have:

(i) $F\left(x_{0}, \ldots, x_{d-2}, x_{d-1}\right)=F\left(x_{0}, \ldots, x_{d-2}\right)$.

(ii) $\operatorname{Gal}(\Omega / F) \simeq \operatorname{Gal}\left(\Omega / F \mathbb{F}_{q}^{\Omega}\right) \simeq(\mathbb{Z} / 2 \mathbb{Z} \times \mathbb{Z} / 2 \mathbb{Z})^{d-1}$.

(iii) The Galois group $\operatorname{Gal}\left(\Omega / \mathbb{F}_{q}(t)\right)$ is an extension of $\mathfrak{S}_{d}$ by $(\mathbb{Z} / 2 \mathbb{Z} \times$ $\mathbb{Z} / 2 \mathbb{Z})^{d-1}$.

(iv) $\Omega / \mathbb{F}_{q}(t)$ is a regular extension i.e. $\mathbb{F}_{q}^{\Omega}=\mathbb{F}_{q}$.

Proof. Suppose that there exists $x \in \mathbb{F}_{q}$ such that $b_{1} / b_{0}=T_{\alpha, \alpha^{\prime}}(x)$. We have $\frac{b_{1}}{b_{0}}=\sum_{i=0}^{d-1} u_{i}=\sum_{i=0}^{d-1} T_{\alpha, \alpha^{\prime}}\left(x_{i}\right)$ and then by linearity we deduce that $T_{\alpha, \alpha^{\prime}}\left(x_{d-1}+x+\sum_{i=0}^{d-2} x_{i}\right)=0$. It implies that $x_{d-1}+x+\sum_{i=0}^{d-2} x_{i} \in$ $\left\{0, \alpha, \alpha^{\prime}, \alpha+\alpha^{\prime}\right\}$ and thus $x_{d-1} \in F\left(x_{0}, \ldots, x_{d-2}\right)$ which proves the point (i). Using (i) and Proposition 4.5 we obtain the point (ii). Now point (ii) with Proposition 3.1 and Galois theory give point (iii). To obtain point (iv), we use the multiplicativity of the degrees in fields extensions and we write $[\Omega: F]=\left[\Omega: F \mathbb{F}_{q}^{\Omega}\right] \times\left[F \mathbb{F}_{q}^{\Omega}: F\right]$. Points (i) and (ii) yield $\left[F \mathbb{F}_{q}^{\Omega}: F\right]=1$ and then the extension $\Omega / F$ is regular. But Proposition 3.1 implies that the extension $F / \mathbb{F}_{q}(t)$ is regular. Then we obtain that the extension $\Omega / \mathbb{F}_{q}(t)$ is regular.

\section{Application of Chebotarev density theorem}

The Chebotarev density theorem describes the proportion of places splitting in a given way in Galois extensions of global fields (see [7] p. 125). In [2, P. Fouque and M. Tibouchi made the following version of Chebotarev theorem explicit. They deduced it from the Proposition 4.6.8 in [3]. 
Theorem 5.1. (Chebotarev) Let $K$ be an extension of $\mathbb{F}_{q}(t)$ of finite degree $d_{K}$ and $L$ a Galois extension of $K$ of finite degree $d_{L / K}$. Assume $\mathbb{F}_{q}$ is algebraically closed in $L$, and fix some subset $S$ of $\mathrm{Gal}(L / K)$ stable under conjugation. Let $s=\sharp S$ and let $N(S)$ be the number of places $v$ of $K$ of degree 1 , unramified in $L$, such that the Artin symbol $\left(\frac{L / K}{v}\right)$ (defined up to conjugation) is in $S$. Then

$\left|N(S)-\frac{s}{d_{L / K}} q\right| \leqslant \frac{2 s}{d_{L / K}}\left(\left(d_{L / K}+g_{L}\right) q^{1 / 2}+d_{L / K}\left(2 g_{K}+1\right) q^{1 / 4}+g_{L}+d_{K} d_{L / K}\right)$

where $g_{K}$ and $g_{L}$ are the genera of the function fields $K$ and $L$.

In this work, we are interested in places of $K=\mathbb{F}_{q}(t)$ which split completely in $L=\Omega$. Indeed, if a place of degree one $(t-\beta)$ with $\beta \in \mathbb{F}_{q}$ totally splits in $\Omega$, then the polynomial $D_{\alpha, \alpha^{\prime}}^{2} f(x)-\beta$ totally splits in $\mathbb{F}_{q}[x]$. These places correspond to places $v$ of $K$ which are unramified in $\Omega$ and for which the Artin symbol $\left(\frac{\Omega / \mathbb{F}_{q}(t)}{v}\right)$ is equal to (id), the conjugacy class of $\operatorname{Gal}\left(\Omega / \mathbb{F}_{q}(t)\right)$ consisting of the identity element. Hence the previous theorem can be used to prove the following proposition which will be the main tool to prove Theorem 7.1 ,

Proposition 5.2. Let $m \geqslant 7$ be an integer and $d$ as defined in Definition 2.1. There exists an integer $N$ depending only on $d$ such that for all $n \geqslant N$, for all $f \in \mathbb{F}_{q}[x]$ (with $q=2^{n}$ ) of degree less or equal to $m$, and for all couple $\left(\alpha, \alpha^{\prime}\right)$ of disctinct elements of $\mathbb{F}_{q}^{*}$ such that the extension $\Omega / \mathbb{F}_{q}(t)$ is regular there exists $\beta \in \mathbb{F}_{q}$ such that the polynomial $D_{\alpha, \alpha^{\prime}}^{2} f(x)+\beta$ splits in $\mathbb{F}_{q}[x]$ with no repeated factors.

Proof. Since the extension $\Omega / \mathbb{F}_{q}(t)$ is regular, by the above Chebotarev theorem the number $N(S)$ of places $v$ of $\mathbb{F}_{q}(t)$ of degree 1 , unramified in $\Omega$, such that $\left(\frac{\Omega / \mathbb{F}_{q}(t)}{v}\right)=($ id) satisfies

$$
N(S) \geqslant \frac{q}{d_{L / K}}-2\left(\left(1+\frac{g_{L}}{d_{L / K}}\right) q^{1 / 2}+q^{1 / 4}+1+\frac{g_{L}}{d_{L / K}}\right) .
$$

From the point ( $i i i)$ of Proposition 4.6 we know that $d_{L / K}=d ! 4^{d-1}$ or $d_{L / K}=d ! 4^{d}$. Moreover, one can obtain an upper bound on $g_{L}$ depending only on $d$ using induction and Castelnuovo's inequality as stated in Theorem 3.11 .3 of [10]. Then if $q$ (or $n$ since $q=2^{n}$ ) is sufficiently large, we will have $N(S) \geqslant 1$, which concludes the proof.

\section{A CLASS OF GOOD POLYNOMIALS}

The last proposition applies when the Galois closure of $D_{\alpha, \alpha^{\prime}}^{2} f-t$ is regular. By Proposition 4.6 this is the case when the quotient of the first coefficients of $L_{\alpha, \alpha^{\prime}}(f)$ can be written in the form $x(x+\alpha)\left(x+\alpha^{\prime}\right)\left(x+\alpha+\alpha^{\prime}\right)$ with $x \in \mathbb{F}_{q}$. Our strategy is now to choose a well fitted finite family $\left(\alpha_{i}, \alpha_{i}^{\prime}\right)_{i \in\{1, \ldots, k\}}$ such that we can apply Proposition 5.2 with at least one couple $\left(\alpha_{i}, \alpha_{i}^{\prime}\right)$ for most of polynomials of degree $m$. 
Proposition 6.1. Let $\varepsilon>0$. There exist $k \in \mathbb{N}^{*}$ and $N \in \mathbb{N}^{*}$ such that for all $n \geqslant N$ there exist $k$ couples $\left(\alpha_{1}, \alpha_{1}^{\prime}\right), \ldots,\left(\alpha_{k}, \alpha_{k}^{\prime}\right)$ of distinct elements of $\mathbb{F}_{q}^{*}$ such that there exist at least $(1-\varepsilon) q^{m}(q-1)-q^{m}$ polynomials $f \in \mathbb{F}_{q}[x]$ of degree $m$ such that:

- for all $i \in\{1, \ldots, k\}$ the polynomial $L_{\alpha_{i}, \alpha_{i}^{\prime}}(f)=b_{0} x^{d}+b_{1} x^{d-1}+\cdots+b_{d}$ has degree $d$ and

- for at least one of the couples $\left(\alpha_{i}, \alpha_{i}^{\prime}\right)$, the equation

$$
\frac{b_{1}}{b_{0}}=x\left(x+\alpha_{i}\right)\left(x+\alpha_{i}^{\prime}\right)\left(x+\alpha_{i}+\alpha_{i}^{\prime}\right)
$$

has a solution in $\mathbb{F}_{q}$.

Proof. Let $f=\sum_{j=0}^{m} a_{j} x^{m-j}$ be a polynomial of degree $m$. First we notice that for any distinct elements $\alpha$ and $\alpha^{\prime}$ of $\mathbb{F}_{q}^{*}$ the polynomial $L_{\alpha, \alpha^{\prime}}(f)$ is of degree $d$ (with $d$ given by Table 1) if and only if $a_{j_{1}} \neq 0$, where $j_{1} \in$ $\{0,1,2,3\}$ is given by Lemma 6.2. In this case, the quotient $b_{1} / b_{0}$ is well defined. By abuse of notation, we will write $\frac{b_{1}}{b_{0}}\left(L_{\alpha, \alpha^{\prime}}(f)\right)$ for this quotient. By linearity of $L_{\alpha, \alpha^{\prime}}$ we have $b_{1} / b_{0}\left(L_{\alpha, \alpha^{\prime}}(\lambda f)\right)=b_{1} / b_{0}\left(L_{\alpha, \alpha^{\prime}}(f)\right)$ for any $\lambda \in \mathbb{F}_{q}^{*}$. So in order to count the polynomials $f$ satisfying the conditions of the proposition we can restrict ourselves to those whose coefficient $a_{j_{1}}$ is 1 , and then multiply by $q-1$ in our count. We will denote by $\mathcal{P}_{j_{1}}$ the set of polynomials $f \in \mathbb{F}_{q}[x]$ of degree $m$ such that $a_{j_{1}}=1$ and we will identify $\mathcal{P}_{j_{1}}$ with $\mathbb{F}_{q}^{m}$.

Let $\varepsilon>0$. Consider $k$ such that $(3 / 4)^{k}<\varepsilon$, and $N=2 k$. For $n \geqslant N$, identify $\mathbb{F}_{2^{n}}$ with $\mathbb{F}_{2}^{n}$ and fix a basis. Consider $k$ couples $\left(\alpha_{1}, \alpha_{1}^{\prime}\right), \ldots,\left(\alpha_{k}, \alpha_{k}^{\prime}\right)$ of distinct elements of $\mathbb{F}_{q}^{*}$ such that for any $i \in\{1, \ldots, k\}$ the subspace $\operatorname{Im} T_{\alpha_{i}, \alpha_{i}^{\prime}}$ has for equation $\left(\xi_{2 i-1}=\xi_{2 i}=0\right)$ in the fixed basis of $\mathbb{F}_{2}^{n}$ (recall that $T_{\alpha, \alpha^{\prime}}$ is defined in Section 4 by $\left.T_{\alpha, \alpha^{\prime}}(x)=x(x+\alpha)\left(x+\alpha^{\prime}\right)\left(x+\alpha+\alpha^{\prime}\right)\right)$. The existence of these couples is given by Lemma 6.3. For any $i \in\{1, \ldots, k\}$ we consider the map $\psi_{i}: \mathcal{P}_{j_{1}} \rightarrow \mathbb{F}_{q}$ defined by $\psi_{i}(f)=b_{1} / b_{0}\left(L_{\alpha_{i}, \alpha_{i}^{\prime}}(f)\right)$. Lemma 6.2 gives the existence of an integer $j_{2}$ (which depends only on the congruence of $m$ ) and the existence of coefficients $c_{i, j}$ and $d_{i}$ in $\mathbb{F}_{q}$ such that

$$
\psi_{i}(f)=a_{j_{2}}+d_{i}+\sum_{j \in\{0, \ldots, m\} \backslash\left\{j_{1}, j_{2}\right\}} c_{i, j} a_{j} .
$$

Now, for $i \in\{1, \ldots, k\}$ the set of $\left(a_{0}, \ldots, a_{j_{1}-1}, a_{j_{1}+1}, \ldots, a_{m}\right) \in \mathbb{F}_{2^{n}}^{m}$ corresponding to elements of $\psi_{i}^{-1}\left(\operatorname{Im} T_{\alpha_{i}, \alpha_{i}^{\prime}}\right)$ is an affine space over $\mathbb{F}_{2}$ which is the intersection of the affine hyperplanes given by the affine equations $\left(a_{j_{2}}\right)_{2 i-1}+\sum_{j \notin\left\{j_{1}, j_{2}\right\}}\left(c_{i, j} a_{j}\right)_{2 i-1}=\left(d_{i}\right)_{2 i-1}$ and $\left(a_{j_{2}}\right)_{2 i}+\sum_{j \notin\left\{j_{1}, j_{2}\right\}}\left(c_{i, j} a_{j}\right)_{2 i}=$ $\left(d_{i}\right)_{2 i}$. The $2 k$ linear forms defined by the left-hand sides of these equations are linearly independant, so a change of basis of the $\mathbb{F}_{2}$-vector space $\mathbb{F}_{2}^{n m}$ gives the following systems of equations of $\psi_{i}^{-1}\left(\operatorname{Im} T_{\alpha_{i}, \alpha_{i}^{\prime}}\right): \zeta_{2 i-1}=\mu_{i}$ and $\zeta_{2 i}=\nu_{i}$ where $\left(\mu_{i}\right)_{i \in\{1, \ldots, k\}}$ and $\left(\nu_{i}\right)_{i \in\{1, \ldots, k\}}$ are elements of $\mathbb{F}_{2}^{k}$. To 
count the elements $\zeta \in \mathbb{F}_{2}^{n m}$ such that $\zeta$ corresponds to an element of $\cup_{i=1}^{k} \psi_{i}^{-1}\left(\operatorname{Im} T_{\alpha_{i}, \alpha_{i}^{\prime}}\right)$ one can determine the cardinal of the complementary. For each $i \in\{1, \ldots, k\}$ there are three ways to choose the couple of components $\left(\zeta_{2 i-1}, \zeta_{2 i}\right)$ different from $\left(\mu_{i}, \nu_{i}\right)$, and $2^{m n-2 k}$ ways to choose the other components.

We find $\# \cup_{i=1}^{k} \psi_{i}^{-1}\left(\operatorname{Im} T_{\alpha_{i}, \alpha_{i}^{\prime}}\right)=2^{m n}-3^{k} 2^{m n-2 k}=q^{m}\left(1-(3 / 4)^{k}\right)$. Finally, we have to multiply by $q-1$ in order to take into account the coefficient $a_{j_{1}}$, and to remove the $q^{m}$ polynomials of degree less than $m$. (Note that in the case where $m \equiv 7 \bmod (8)$ we have already removed these polynomials as we have supposed $a_{j_{1}} \neq 0$ and in this case $j_{1}=0$.)

Lemma 6.2. Let $f=\sum_{j=0}^{m} a_{j} x^{m-j}$ be a polynomial of $\mathbb{F}_{q}[x]$ of degree $m$ with $m \equiv 0,1,2$ or $7(\bmod 8)$. For $\alpha, \alpha^{\prime} \in \mathbb{F}_{q}^{*}$ we set $L_{\alpha, \alpha^{\prime}}(f)=$ $\sum_{j=0}^{d} b_{j} x^{d-j}$. We have $b_{0}=\alpha \alpha^{\prime}\left(\alpha+\alpha^{\prime}\right) a_{i}$ where $i \in\{0,1,2,3\}$ satisfies $i \equiv m+1 \bmod 4$. Moreover the following table gives the quotient $b_{1} / b_{0}$ as a function of the coefficients of $f$ depending on the congruence of m modulo 16.

\begin{tabular}{|c|c|}
\hline$m$ & $b_{1} / b_{0}$ \\
$(16)$ & $\left(\left(\alpha^{2} \alpha^{\prime}+\alpha^{\prime 2} \alpha\right) a_{2}+\left(\alpha^{2}+\alpha \alpha^{\prime}+\alpha^{\prime 2}\right) a_{3}+a_{5}\right) a_{1}^{-1}$ \\
\hline 0 & $\left(\left(\alpha^{2} \alpha^{\prime}+\alpha^{\prime 2} \alpha\right) a_{3}+\left(\alpha^{2}+\alpha \alpha^{\prime}+\alpha^{\prime 2}\right) a_{4}+a_{6}\right) a_{2}^{-1}$ \\
1 & $\left(\left(\alpha^{2} \alpha^{\prime}+\alpha^{\prime 2} \alpha\right) a_{4}+\left(\alpha^{2}+\alpha \alpha^{\prime}+\alpha^{\prime 2}\right) a_{5}+a_{7}\right) a_{3}^{-1}$ \\
2 & $\left(\left(\alpha^{2} \alpha^{\prime}+\alpha^{\prime 2} \alpha\right) a_{1}+\left(\alpha^{2}+\alpha \alpha^{\prime}+\alpha^{\prime 2}\right) a_{2}+a_{4}\right) a_{0}^{-1}+\alpha^{4}+\alpha^{2} \alpha^{\prime 2}+\alpha^{\prime 4}$ \\
7 & $\left(\left(\alpha^{2} \alpha^{\prime}+\alpha^{\prime 2} \alpha\right) a_{2}+\left(\alpha^{2}+\alpha \alpha^{\prime}+\alpha^{\prime 2}\right) a_{3}+a_{5}\right) a_{1}^{-1}+\alpha^{4}+\alpha^{2} \alpha^{\prime 2}+\alpha^{\prime 4}$ \\
8 & $\left(\sum_{i=0}^{6} \alpha^{i} \alpha^{\prime 6-i} a_{0}+\left(\alpha^{2} \alpha^{\prime}+\alpha^{\prime 2} \alpha\right) a_{3}+\left(\alpha^{2}+\alpha \alpha^{\prime}+\alpha^{\prime 2}\right) a_{4}+a_{6}\right) a_{2}^{-1}+\alpha^{4}+\alpha^{2} \alpha^{\prime 2}+\alpha^{\prime 4}$ \\
9 & $\left(a_{0} \sum_{i=1}^{6} \alpha^{i} \alpha^{\prime 7-i}+a_{1} \sum_{i=0}^{6} \alpha^{i} \alpha^{\prime 6-i}+\left(\alpha^{2} \alpha^{\prime}+\alpha^{\prime 2} \alpha\right) a_{4}+\left(\alpha^{2}+\alpha \alpha^{\prime}+\alpha^{\prime 2}\right) a_{5}+a_{7}\right) a_{3}^{-1}$ \\
10 & $+\alpha^{4}+\alpha^{2} \alpha^{\prime 2}+\alpha^{\prime 4}$ \\
15 & $\left(\left(\alpha^{2} \alpha^{\prime}+\alpha^{\prime 2} \alpha\right) a_{1}+\left(\alpha^{2}+\alpha \alpha^{\prime}+\alpha^{\prime 2}\right) a_{2}+a_{4}\right) a_{0}^{-1}$ \\
\hline
\end{tabular}

Proof. The question amounts to solving the linear system

$$
\sum_{j=0}^{d} b_{j}\left(x(x+\alpha)\left(x+\alpha^{\prime}\right)\left(x+\alpha+\alpha^{\prime}\right)\right)^{d-j}=D_{\alpha, \alpha^{\prime}}^{2}\left(\sum_{j=0}^{m} a_{j} x^{m-j}\right) .
$$

On the one hand we have

$$
D_{\alpha, \alpha^{\prime}}^{2} f(x)=\sum_{j=1}^{m}\left(\sum_{s=1}^{j} a_{j-s} C_{s}\left(\begin{array}{c}
m-j+s \\
s
\end{array}\right)\right) x^{m-j}
$$


where $C_{s}$ denotes $\alpha^{s}+\alpha^{s}+\left(\alpha+\alpha^{\prime}\right)^{s}$ for $s \geqslant 1$. We notice that $C_{1}=C_{2}=$ $C_{4}=0$ and that $C_{3}=\alpha \alpha^{\prime}\left(\alpha+\alpha^{\prime}\right)$. It implies

$$
\begin{aligned}
D_{\alpha, \alpha^{\prime}}^{2} f(x)= & \left(\begin{array}{c}
m \\
3
\end{array}\right) a_{0} C_{3} x^{m-3}+\left(\begin{array}{c}
m-1 \\
3
\end{array}\right) a_{1} C_{3} x^{m-4} \\
& +\left(\left(\begin{array}{c}
m \\
5
\end{array}\right) a_{0} C_{5}+\left(\begin{array}{c}
m-2 \\
3
\end{array}\right) a_{2} C_{3}\right) x^{m-5} \\
& +\left(\left(\begin{array}{c}
m \\
6
\end{array}\right) a_{0} C_{6}+\left(\begin{array}{c}
m-1 \\
5
\end{array}\right) a_{1} C_{5}+\left(\begin{array}{c}
m-3 \\
3
\end{array}\right) a_{3} C_{3}\right) x^{m-6}+\cdots
\end{aligned}
$$

On the other hand, the left-hand side of (2) is equal to

$$
\begin{array}{r}
g\left(T_{\alpha, \alpha^{\prime}}(x)\right)=b_{0} x^{4 d}+b_{0} d\left(\alpha^{2}+\alpha^{\prime 2}+\alpha \alpha^{\prime}\right) x^{4 d-2}+b_{0} d\left(\alpha+\alpha^{\prime}\right) \alpha \alpha^{\prime} x^{4 d-3} \\
+\left(b_{0}\left(\begin{array}{l}
d \\
2
\end{array}\right)\left(\alpha^{2}+\alpha^{\prime 2}+\alpha \alpha^{\prime}\right)^{2}+b_{1}\right) x^{4 d-4}+\cdots
\end{array}
$$

To obtain $b_{0}$ (and respectively $b_{1}$ ) one can identify the coefficients of $x^{4 d}$ (respectively $x^{4 d-4}$ ) on both sides of (2). To distinguish different cases and conclude we use a classical consequence of Lucas's theorem which says that a binomial coefficient $\left(\begin{array}{l}a \\ b\end{array}\right)$ is divisible by 2 if and only if at least one of the base 2 digits of $b$ is greater than the corresponding digit of $a$.

We use the following representation lemma as a key point in the proof of Proposition 6.1.

Lemma 6.3. Let $V$ be a $\mathbb{F}_{2}$-vectorial subspace of $\mathbb{F}_{q}$ of codimension 2 . Then there exist two distincts elements $\alpha$ and $\alpha^{\prime}$ in $\mathbb{F}_{q}^{*}$ such that $V=\operatorname{Im} T_{\alpha, \alpha^{\prime}}$ where $T_{\alpha, \alpha^{\prime}}(x)=x(x+\alpha)\left(x+\alpha^{\prime}\right)\left(x+\alpha+\alpha^{\prime}\right)$.

Proof. First we prove that $\operatorname{Im} T_{\alpha, \alpha^{\prime}}$ is the intersection of the kernels of the morphisms $x \mapsto \operatorname{Tr}_{\mathbb{F}_{2^{n}} / \mathbb{F}_{2}}\left(\frac{x}{\left(\alpha^{2}+\alpha \alpha^{\prime}\right)^{2}}\right)$ and $x \mapsto \operatorname{Tr}_{\mathbb{F}_{2^{n}} / \mathbb{F}_{2}}\left(\frac{x}{\left(\alpha^{\prime 2}+\alpha \alpha^{\prime}\right)^{2}}\right)$ where $\operatorname{Tr}_{\mathbb{F}_{2^{n} / \mathbb{F}_{2}}}$ is the Trace function relative to the extension $\mathbb{F}_{2^{n}} / \mathbb{F}_{2}$. Let us prove that $\operatorname{Im} T_{\alpha, \alpha^{\prime}}$ is included in the kernel of one the two morphisms. Indeed, if $z=T_{\alpha, \alpha^{\prime}}(x)$, then $z=u(u+\gamma)$ with $\gamma=\alpha^{\prime 2}+\alpha \alpha^{\prime}$ and $u=x(x+\alpha)$. The Hilbert 90 Theorem implies that $\operatorname{Tr}_{\mathbb{F}_{2 n} / \mathbb{F}_{2}}\left(z / \gamma^{2}\right)=0$ and we are done. We have the inclusion in the kernel of the other morphism by symmetry, and we conclude with a dimension argument.

As any hyperplane of $\mathbb{F}_{2^{n}}$ is the kernel of a linear form $x \mapsto \operatorname{Tr}(w \cdot x)$ for a good choice of $w \in \mathbb{F}_{2^{n}}^{*}$, and as $x \mapsto 1 / x^{2}$ is a bijection onto $\mathbb{F}_{2^{n}}^{*}$ it is now sufficient to prove that for all couple $(u, v)$ of distinct elements of $\mathbb{F}_{2^{n}}^{*}$ there exists a couple of distinct elements $\left(\alpha, \alpha^{\prime}\right)$ of $\mathbb{F}_{2^{n}}^{*}$ such that $\alpha^{2}+\alpha \alpha^{\prime}=u$ and $\alpha^{\prime 2}+\alpha \alpha^{\prime}=v$. To this end, we consider the function $\Theta$ : $\mathbb{F}_{2^{n}}^{*} \times \mathbb{F}_{2^{n}}^{*} \backslash \Delta \rightarrow \mathbb{F}_{2^{n}}^{*} \times \mathbb{F}_{2^{n}}^{*} \backslash \Delta$ which maps $\left(\alpha, \alpha^{\prime}\right)$ to $\left(\alpha^{2}+\alpha \alpha^{\prime}, \alpha^{\prime 2}+\alpha \alpha^{\prime}\right)$ where $\Delta$ denotes the diagonal. It is well defined because if $\alpha^{2}+\alpha \alpha^{\prime}=\alpha^{\prime 2}+\alpha \alpha^{\prime}$ then $\alpha^{2}=\alpha^{\prime 2}$ and so $\alpha=\alpha^{\prime}$. If $\Theta\left(\alpha_{1}, \alpha_{1}^{\prime}\right)=\Theta\left(\alpha_{2}, \alpha_{2}^{\prime}\right)$, then one has the two equalities $\alpha_{1}^{2}+\alpha_{1} \alpha_{1}^{\prime}=\alpha_{2}^{2}+\alpha_{2} \alpha_{2}^{\prime}$ and $\alpha_{1}^{\prime 2}+\alpha_{1} \alpha_{1}^{\prime}=\alpha_{2}^{\prime 2}+\alpha_{2} \alpha_{2}^{\prime}$. 
It implies $\left(\alpha_{1}+\alpha_{1}^{\prime}\right)^{2}=\left(\alpha_{2}+\alpha_{2}^{\prime}\right)^{2}$ and so there exists $\mu \in \mathbb{F}_{2^{n}}$ such that $\mu=\alpha_{1}+\alpha_{1}^{\prime}=\alpha_{2}+\alpha_{2}^{\prime}$. Using the first equality one obtains $\alpha_{1} \mu=\alpha_{2} \mu$. We know that $\mu \neq 0$, otherwise we would have $\alpha_{1}=\alpha_{1}^{\prime}$, and $\left(\alpha_{1}, \alpha_{1}^{\prime}\right) \in \Delta$, a contradiction. So we can deduce $\alpha_{1}=\alpha_{2}$ and using the first equality one more time we have $\alpha_{1} \alpha_{1}^{\prime}=\alpha_{2} \alpha_{2}^{\prime}$, and so $\alpha_{1}^{\prime}=\alpha_{2}^{\prime}$. Hence the function $\Theta$ is injective and thus bijective.

\section{MAIN THEOREM}

We will use all the previous propositions to prove our main result, namely that most polynomials $f$ over $\mathbb{F}_{q}$ have a maximal $\delta^{2}(f)$. More precisely, we prove the following theorem.

Theorem 7.1. Let $m$ be an integer such that $m \geqslant 7$ and $m \equiv 0(\bmod 8)$ (respectively $m \equiv 1,2,7(\bmod 8))$, let $\delta_{0}=m-4$ (respectively $\delta_{0}=m-$ $5, m-6, m-3)$. Then we have

$$
\lim _{n \rightarrow \infty} \frac{\sharp\left\{f \in \mathbb{F}_{2^{n}}[x] \mid \operatorname{deg}(f)=m, \delta^{2}(f)=\delta_{0}\right\}}{\sharp\left\{f \in \mathbb{F}_{2^{n}}[x] \mid \operatorname{deg}(f)=m\right\}}=1 .
$$

Proof. Recall that we set $q=2^{n}$. We fix an integer $m \geqslant 7$ and consequently an integer $d$ defined by Table 1 and an integer $\tilde{d}$ depending only on $d$ as introduced in Proposition 3.2 .

Let $\varepsilon>0$. We fix an integer $N_{1}$ satisfying the properties of Proposition 5.2. By Proposition 6.1 there exist integers $k$ and $N_{2}$ such that for any $n \geqslant N_{2}$ we can choose $k$ couples $\left(\alpha_{1}, \alpha_{1}^{\prime}\right), \ldots,\left(\alpha_{k}, \alpha_{k}^{\prime}\right)$ of disctinct elements of $\mathbb{F}_{q}^{*}$ such that for at least $(1-\varepsilon)(q-1) q^{m}-q^{m}$ polynomials $f \in \mathbb{F}_{q}[x]$ of degree $m$ the polynomial $L_{\alpha_{i}, \alpha_{i}^{\prime}}(f)$ has degree $d$ for all $i$, and at least one of the $k$ equations

$$
\frac{b_{1}}{b_{0}}=x\left(x+\alpha_{i}\right)\left(x+\alpha_{i}^{\prime}\right)\left(x+\alpha_{i}+\alpha_{i}^{\prime}\right)
$$

has a solution in $\mathbb{F}_{q}$, where $L_{\alpha_{i}, \alpha_{i}^{\prime}}(f(x))=b_{0} x^{d}+b_{1} x^{d-1}+\cdots+b_{d}$. Finally, we fix an integer $N_{3}$ such that for all $n \geqslant N_{3}$

$$
0 \leqslant \frac{q^{m}+k \tilde{d} q^{m}}{(q-1) q^{m}} \leqslant \varepsilon .
$$

Let $n \geqslant \operatorname{Max}\left(N_{1}, N_{2}, N_{3}\right)$ and a polynomial $f$ associated to a couple $\left(\alpha_{i}, \alpha_{i}^{\prime}\right)$ satisfying the preceeding conditions. If we suppose that $L_{\alpha_{i}, \alpha_{i}^{\prime}}(f)$ is Morse, then by Proposition 4.6 the extension $\Omega / \mathbb{F}_{q}(t)$ is regular where $\Omega$ is the Galois closure of $D_{\alpha_{i}, \alpha_{i}^{\prime}} f(x)+t$. Hence by Proposition 5.2 there exists $\beta \in \mathbb{F}_{q}$ such that $D_{\alpha_{i}, \alpha_{i}^{\prime}}^{2}(f)(x)=\beta$ has $4 d$ solutions in $\mathbb{F}_{q}$. It amounts to saying that $\delta^{2}(f)=\delta_{0}$. Let us count these polynomials: $f$ is choosen among the $(1-\varepsilon)(q-1) q^{m}-q^{m}$ polynomials given by Proposition 6.1, but we have to remove the polynomials $f$ such that for all $i \in\{1, \ldots, k\}$ the polynomial $L_{\alpha_{i}, \alpha_{i}^{\prime}}(f)$ is non-Morse. Thanks to Proposition 3.2 we know we have to remove at most $k \tilde{d} q^{m}$ polynomials. To obtain the density we have to divide 
by $(q-1) q^{m}$ which is the number of polynomials of degree $m$. Finally, the condition (3) above ensures that this density is greater than or equal to $1-2 \varepsilon$.

\section{The INVERSiOn MAPPING}

We conclude the paper by the study of the second order differential uniformity of the inversion mapping from $\mathbb{F}_{q}$ (with $q=2^{n}$ ) to itself which sends $x$ to $x^{-1}$ if $x \neq 0$ and 0 to 0 and which corresponds to the polynomial $f(x)=x^{q-2}$ of $\mathbb{F}_{q}[x]$. The $S$-box used by AES involves precisely this function in the case where $n=8$. Nyberg proved in [6] that it has a differential uniformity $\delta(f)=2$ for $n$ odd and $\delta(f)=4$ for $n$ even.

We determine here its second order differential uniformity over $\mathbb{F}_{2^{n}}$ for any $n$. By a direct computation, we can show that $\delta^{2}(f)=4$ over $\mathbb{F}_{2^{n}}$ for $n=2,4$ and 5 and that $\delta^{2}(f)=8$ for $n=3$. For $n \geqslant 6$, we have the following proposition.

Proposition 8.1. The inversion mapping $f$ over $\mathbb{F}_{2^{n}}$ has a second order differential uniformity $\delta^{2}(f)=8$ for any $n \geqslant 6$.

Proof. Set $q=2^{n}$ and let $\alpha, \alpha^{\prime} \in \mathbb{F}_{q}^{*}$ such that $\alpha \neq \alpha^{\prime}$ and $\beta \in \mathbb{F}_{q}$. Consider the equation $D_{\alpha, \alpha^{\prime}} f(x)=\beta$ i.e.

$$
x^{q-2}+(x+\alpha)^{q-2}+\left(x+\alpha^{\prime}\right)^{q-2}+\left(x+\alpha+\alpha^{\prime}\right)^{q-2}=\beta .
$$

Since $f$ is a monomial function, this equation can be written:

$\alpha^{\prime q-2}\left(\left(\frac{x}{\alpha^{\prime}}\right)^{q-2}+\left(\frac{x}{\alpha^{\prime}}+\frac{\alpha}{\alpha^{\prime}}\right)^{q-2}+\left(\frac{x}{\alpha^{\prime}}+1\right)^{q-2}+\left(\frac{x}{\alpha^{\prime}}+\frac{\alpha}{\alpha^{\prime}}+1\right)^{q-2}\right)=\beta$.

Thus in order to compute $\delta^{2}(f)$ we can suppose that $\alpha^{\prime}=1$. So we consider now for $\alpha \in \mathbb{F}_{q} \backslash\{0,1\}$ and $\beta \in \mathbb{F}_{q}$ the number of solutions of the equation:

$$
x^{q-2}+(x+\alpha)^{q-2}+(x+1)^{q-2}+(x+\alpha+1)^{q-2}=\beta .
$$

If $x \notin\{0,1, \alpha, \alpha+1\}$, then this equation is equivalent to:

$$
x^{-1}+(x+\alpha)^{-1}+(x+1)^{-1}+(x+\alpha+1)^{-1}=\beta
$$

which is equivalent to:

$$
\beta T_{\alpha, 1}(x)+\alpha(\alpha+1)=0
$$

where $T_{\alpha, \alpha^{\prime}}(x)=x(x+\alpha)\left(x+\alpha^{\prime}\right)\left(x+\alpha+\alpha^{\prime}\right)$ as introduced in Section 4 .

Thus Equation (5D) has at most four solutions in $\mathbb{F}_{q} \backslash\{0,1, \alpha, \alpha+1\}$. Precisely, it has no solution or it has four solutions since $T_{\alpha, 1}(x)=T_{\alpha, 1}(x+$ $\alpha)=T_{\alpha},(x+1)=T_{\alpha, 1}(x+\alpha+1)$.

An element $x \in\{0,1, \alpha, \alpha+1\}$ is a solution of Equation (4) if and only if $\beta=\frac{\alpha^{2}+\alpha+1}{\alpha(\alpha+1)}$. Now let us solve Equation (4) in $\mathbb{F}_{q} \backslash\{0,1, \alpha, \alpha+1\}$ with such $\beta$. If $\beta=0$ then Equation (5) has no solution so we can suppose that $\beta \neq 0$ i.e. $\alpha^{2}+\alpha+1 \neq 0$. Then equation (5) can be written $T_{\alpha, 1}(x)=$ 
$\gamma$ where $\gamma=\frac{\alpha^{2}\left(\alpha^{2}+1\right)}{\alpha^{2}+\alpha+1}$. We have shown in the proof of Lemma 6.3 that $\operatorname{Im} T_{\alpha, \alpha^{\prime}}$ is equal to the intersection of the kernels of the morphisms $x \mapsto$ $\operatorname{Tr}_{\mathbb{F}_{2^{n}} / \mathbb{F}_{2}}\left(\frac{x}{\left(\alpha^{2}+\alpha \alpha^{\prime}\right)^{2}}\right)$ and $x \mapsto \operatorname{Tr}_{\mathbb{F}_{2^{n}} / \mathbb{F}_{2}}\left(\frac{x}{\left(\alpha^{\prime 2}+\alpha \alpha^{\prime}\right)^{2}}\right)$. Hence the equation $T_{\alpha, 1}(x)=\gamma$ has a solution if and only if $\gamma$ is in the intersection of the kernels of these two maps, i.e.

$$
\operatorname{Tr}_{\mathbb{F}_{2^{n}} / \mathbb{F}_{2}}\left(\frac{1}{\alpha^{2}+\alpha+1}\right)=0 \text { and } \operatorname{Tr}_{\mathbb{F}_{2^{n}} / \mathbb{F}_{2}}\left(\frac{\alpha^{2}}{\alpha^{2}+\alpha+1}\right)=0 .
$$

In the case where $n$ is even, any element in the subfield $\mathbb{F}_{2^{n / 2}}$ has a trace equal to zero. Thus, any $\alpha$ different from 0 and 1 in this subfield and with $\alpha^{2}+\alpha+1 \neq 0$ verifies the two previous conditions of (6). Thus if the subfield $\mathbb{F}_{2^{n / 2}}$ have more than 4 elements, i.e. if $n>4$ then $\delta^{2}(f)=8$.

In order to solve the problem in the case where $n$ is odd, consider the algebraic surfaces $S_{1}$ and $S_{2}$ in the affine space $\mathbb{A}^{3}$ given respectively by the equations $\left(y^{2}+y\right)\left(x^{2}+x+1\right)=1$ and $\left(z^{2}+z\right)\left(x^{2}+x+1\right)=x^{2}$. Consider the affine curve $C=S_{1} \cap S_{2}$ in $\mathbb{A}^{3}$. By Hilbert 90 theorem, a solution $\alpha$ in $\mathbb{F}_{2^{n}}$ to Equations (6) corresponds to four points $(x, y, z)$ on $C$.

Furthermore if $(x, y, z) \in C$ then we can show that $x\left(y^{2}+y\right)+y^{2}+y+$ $z^{2}+z+1=0$ and $x\left(z^{2}+z+1\right)+y^{2}+y+1=0$. Then we obtain:

$$
\left(y^{2}+y\right)^{2}+\left(y^{2}+y\right)\left(z^{2}+z\right)+\left(z^{2}+z+1\right)^{2}=0 .
$$

Consider the projection

$$
\pi: \begin{array}{ccc}
\mathbb{A}^{3} & \longrightarrow \mathbb{A}^{2} \\
(x, y, z) & \longmapsto(y, z)
\end{array}
$$

and the affine plane curve $D$ defined by Equation (7). Consider also $Z=$ $\left\{(y, z) \in \mathbb{A}^{2} \mid y^{2}+y=0\right.$ and $\left.z^{2}+z+1=0\right\}$. The set $Z$ has 4 points and each of them has degree 2 over $\mathbb{F}_{2}$. The projection $\pi$ provides an isomorphism between $C$ and $D \backslash Z$ whose inverse is given by:

$$
\begin{aligned}
& D \backslash Z \rightarrow C \\
& (y, z) \mapsto \begin{cases}\left(\frac{z^{2}+z+1}{y^{2}+y}+1, y, z\right) & \text { if } y^{2}+y \neq 0, \\
\left(\frac{y^{2}+y+1}{z^{2}+z+1}, y, z\right) & \text { if } z^{2}+z+1 \neq 0 .\end{cases}
\end{aligned}
$$

Let us denote by $\bar{D}$ the projective closure of $D$ in the projective plane $\mathbb{P}^{2}$. It has 2 points at infinity and each of them has degree 2 .

It follows that the curves $C$ and $\bar{D}$ have the same number of rational points over $\mathbb{F}_{2^{n}}$ for $n$ odd. Furthermore, the curve $\bar{D}$ is a smooth projective 
plane quartic, so it is absolutely irreducible and has genus 3. By Serre-Weil theorem (see [8]), the number of rational points over $\mathbb{F}_{2^{n}}$ of $\bar{D}$ verifies:

$$
\sharp \bar{D}\left(\mathbb{F}_{2^{n}}\right) \geqslant 2^{n}+1-3\left[2^{(n+2) / 2}\right] .
$$

So, if $n \geqslant 7$, we have $\sharp C\left(\mathbb{F}_{2^{n}}\right) \geqslant 63$ and then there are at least 15 solutions to Equations (6) and the result follows.

Acknowledgments: The authors want to thank Felipe Voloch for lightning discussions, particularly concerning the strategy described in Section 6. They also want to thank Philippe Langevin, René Schoof and David

Kohel for a nice discussion concerning the last section and the referee for helpful comments.

\section{References:}

\section{REFERENCES}

[1] A. I. Borevich and I. R. Shafarevich. Number theory. Translated from the Russian by Newcomb Greenleaf. Pure and Applied Mathematics, Vol. 20. Academic Press, New York-London, 1966.

[2] Pierre-Alain Fouque and Mehdi Tibouchi. Estimating the size of the image of deterministic hash functions to elliptic curves. In Progress in Cryptology - Latincrypt 2010, volume 6212 of Lecture Notes in Computer Science, pages 81-91, 2010.

[3] Michael D. Fried and Moshe Jarden. Field arithmetic, volume 11 of Ergebnisse der Mathematik und ihrer Grenzgebiete. 3. Folge. A Series of Modern Surveys in Mathematics. Springer-Verlag, Berlin, second edition, 2005.

[4] Moshe Jarden and Aharon Razon. Skolem density problems over large Galois extensions of global fields. In Hilbert's tenth problem: relations with arithmetic and algebraic geometry (Ghent, 1999), volume 270 of Contemp. Math., pages 213-235. Amer. Math. Soc., Providence, RI, 2000. With an appendix by Wulf-Dieter Geyer.

[5] Xuejia Lai. Higher order derivatives and differential cryptanalysis. In Communications and Cryptography, pages 227-233. Springer, 1994.

[6] Kaisa Nyberg. Differentially uniform mappings for cryptography. In Advances in cryptology-Eurocrypt '93, volume 765 of Lecture Notes in Computer Science, pages 55-64. Springer, Berlin, 1994.

[7] Michael Rosen. Number theory in function fields, volume 210 of Graduate Texts in Mathematics. Springer-Verlag, New York, 2002.

[8] Jean-Pierre Serre. Sur le nombre des points rationnels d'une courbe algébrique sur un corps fini. C. R. Acad. Sci. Paris Sér. I Math., 296(9):397-402, 1983.

[9] Jean-Pierre Serre. Topics in Galois theory, volume 1 of Research Notes in Mathematics. A K Peters, Ltd., Wellesley, MA, second edition, 2008. With notes by Henri Darmon.

[10] Henning Stichtenoth. Algebraic function fields and codes, volume 254 of Graduate Texts in Mathematics. Springer-Verlag, Berlin, second edition, 2009.

[11] José Felipe Voloch. Symmetric cryptography and algebraic curves. In Algebraic geometry and its applications, volume 5 of Ser. Number Theory Appl., pages 135-141. World Sci. Publ., Hackensack, NJ, 2008. 
(Yves Aubry) Institut de Mathématiques de Toulon, Université de Toulon, FRANCE

(Yves Aubry) Aix-Marseille Univ, CNRS, Centrale Marseille, I2M, MarSEILle, FRANCE

E-mail address: yves.aubry@univ-tln.fr

(Fabien Herbaut) Institut de Mathématiques De Toulon, Université De Toulon, FRANCE

(Fabien Herbaut) ESPE Nice-Toulon, Université Nice Sophia Antipolis, France

E-mail address: fabien.herbaut@unice.fr 